\title{
Sonication-Induced Scission of Molecular Bottlebrushes: Implications of the "Hairy" Architecture
}

Yuanchao Li ${ }^{\text {a }}$, Zhenbin Niu ${ }^{b}$, Joanna Burdyńska ${ }^{c}$, Alper Nese ${ }^{c}$, Yang Zhou ${ }^{a}$, Zachary S. Kean

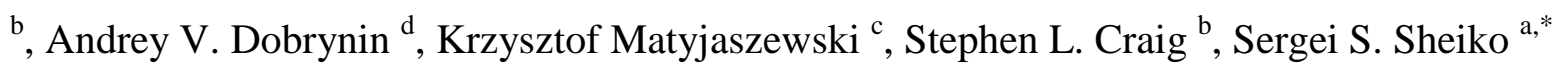

${ }^{a}$ Department of Chemistry, University of North Carolina, Chapel Hill, North Carolina 27599, United States

${ }^{\mathrm{b}}$ Department of Chemistry, Duke University, Durham, North Carolina 27708, United States

${ }^{\mathrm{c}}$ Department of Chemistry, Carnegie Mellon University, Pittsburgh, Pennsylvania 15213, United States

${ }^{\mathrm{d}}$ Department of Polymer Science, University of Akron, Akron, Ohio 44325, United States

* Correspondence to: Sergei S. Sheiko (Email: sergei@email.unc.edu) 


\begin{abstract}
Polymer bottlebrushes may be viewed as hairy flexible cylinders with a long backbone and a thick corona of densely grafted polymer chains. The corona not only controls the bottlebrush diameter, but also provides an additional drag force. We report the study of backbone scission by external forces caused by ultrasonication. A series of bottlebrushes with the same backbone and different side-chain lengths was prepared by ATRP. Bond fracture was induced by pulsed ultrasound in a dilute chloroform solution and ex-situ monitored through molecular imaging of reaction products by atomic force microscopy. The scission rate was found to increase with side chain length, while the limiting length of fractured bottlebrushes displayed a decrease. The experiment showed a good agreement with the Rouse model of polymer dynamics, which suggests that solvent drains through the corona of bottlebrush side-chains.
\end{abstract}




\section{Introduction}

Molecular bottlebrushes are a unique class of graft copolymers exhibiting extended comblike conformation due to steric repulsion of densely grafted side chains [1,2]. The special spatial dimensions and tunable architecture of bottlebrush macromolecules lead to potential applications including supersoft elastomers [3-6], nanomaterials fabrication [7, 8] and molecular tensile machines [9-13]. We have been particularly interested in the use of bottlebrushes as molecular tensile machines where tension is self-generated along the backbone due to steric repulsion between densely grafted side chains $[14,15]$. The intrinsic tension can be finely tuned by adjusting molecular architecture (side chain length and grafting density) and environmental conditions (solvent quality and temperature), enabling mechanical activation of intramolecular chemical reactions in response to minute variations in temperature [11], surface energy [16], and concentration of reducing agents [17]. In addition to the internal tension, the bottlebrush architecture may effectively mediate the effect of externally applied forces, e.g. during shear flow upon sonication-induced solvent cavitation. Nowadays, sonication is widely used in the dispersion of nano- and mesoscale particles and filaments. In addition, it can also break carbon nanotubes [18-21], cylindrical micelles [22-24], and polymer fibers [25, 26]. It is generally accepted that the destructive force is caused by ultrafast shear flow of the solvent created by cavitation, which involves the nucleation, growth, and collapse of microbubbles in solution. As such, sonication has become a popular technique to control the length or measure the mechanical strength of nanostructured materials, including carbon nanotubes, nanowires, cylindrical micelles and fibrils [19, 27, 28].

In this work, we study the ultrasound-induced scission of molecular bottlebrushes in dilute chloroform solutions, which could be the result of contributions from both intrinsic and external forces. Unlike solid cylindrically shaped objects, molecular bottlebrushes are "soft" and their 
hydrodynamic diameters depend on both side-chain degree of polymerization (DP) and solvent quality. Here, we are particularly interested in the effects of the side chain DP on the sonicationinduced backbone scission, including the scission rate and the limiting length of the fracture products. The side-chain effect depends on the sonication-induced flow rate, which may result in solvent draining through the bottlebrush corona producing an additional drag force and leading to amplification of the scission process. As a result, the contribution of polymer architecture to sonication induced chain fracture is expected to be much richer than has been observed, for example, in three-arm star polymers [29]. Molecular bottlebrushes prepared from the same backbone with different side chain DPs were subjected to pulsed ultrasound to cause backbone scission. The scission kinetics was followed by monitoring the average contour length of the bottlebrushes via molecular imaging with the use of atomic force microscopy (AFM). We have analyzed the data and shown the dependence of the limiting contour length of the bottlebrush backbone and the scission rate on the side chain DP.

\section{Experimental Section}

2.1 Materials. $n$-Butyl acrylate ( $n \mathrm{BA}, 99 \%$, Acros) and (2-trimetylsiloxy)ethyl methacrylate (HEMA-TMS, Scientific Polymer Products) were purified by passing the monomer through a column filled with basic alumina to remove the inhibitor, 2,2'-azobis(2-methylpropionitrile) (AIBN, 98\%, Aldrich) was recrystallized from methanol and dried under vacuum prior use. Sulfuric acid (20\% fuming) was purchased from Alfa Aesar. All other reagents: 2-cyano-2propyl 4-cyanobenzodithioate (98\%), copper(I) bromide ( $\left.\mathrm{Cu}{ }^{\mathrm{I}} \mathrm{Br}, 99.999 \%\right)$, copper(II) bromide $\left(\mathrm{Cu}^{\mathrm{II}} \mathrm{Br}_{2}, 99.999 \%\right), 4,4^{\prime}$-dinonyl-2,2'-dipyridyl (dNbpy, 97\%), potassium fluoride (KF, 99\%), tetrabutylammonium fluoride (TBAF, 1.0 M in THF), $\alpha$-bromoisobutyryl bromide (98\%), 2,5-di- 
tert-butylphenol (DTBP, 99\%), triethylamine (TEA, $\leq 99 \%$ ), 1-butanol (ACS reagent, $\geq 99.4 \%$ ) and solvents were purchased from Aldrich and used as received without further purification.

\subsection{Synthesis and Characterization}

The conversion of $n \mathrm{BA}$ was determined from ${ }^{1} \mathrm{H}$ NMR spectra recorded in $\mathrm{CDCl}_{3}$ as a solvent using Brüker $300 \mathrm{MHz}$ spectrometer. Molecular weight distributions of the polymers were characterized by gel permeation chromatography (GPC) using Polymer Standards Services (PSS) columns (guard, $10^{5}, 10^{3}$, and $10^{2} \AA$ ), with THF eluent at $35^{\circ} \mathrm{C}$, flow rate $1.00 \mathrm{~mL} / \mathrm{min}$, and differential refractive index (RI) detector (Waters, 2410). The apparent number-average molecular weights $\left(M_{\mathrm{n}}\right)$ and molecular weight dispersities $\left(M_{\mathrm{w}} / M_{\mathrm{n}}\right)$ were determined with a calibration based on linear poly(methyl methacrylate) (PMMA) standards and diphenyl ether as an internal standard, using WinGPC 6.0 software from PSS. In addition to the conventional GPC technique, we used a combination of molecular imaging by AFM and preparation of monolayers at a controlled mass-per-unit-area by the Langmuir-Blodgett technique (LB). While the GPC of large branched macromolecules is prone to significant errors, the AFM-LB method provides more accurate data on the DP of both the backbone and side chains [30, 31].

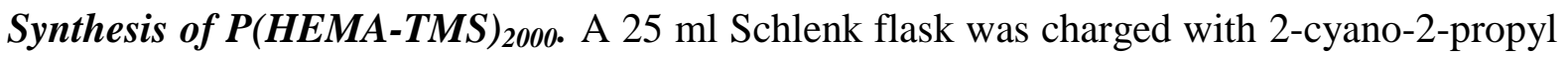
4-cyanobenzodithioate $(0.0023 \mathrm{~g}, 0.0092 \mathrm{mmol})$, HEMA-TMS (20.0 mL, $91.9 \mathrm{mmol})$, AIBN (0.151 mg, $0.92 \mu \mathrm{mol}$, a stock solution) and toluene $(1.0 \mathrm{~mL})$. The solution was degassed by purging with nitrogen over $30 \mathrm{~min}$. Afterwards, the sealed flask was immersed in an oil bath at $65^{\circ} \mathrm{C}$. Polymerization was terminated after $65 \mathrm{~h}$ at $20.5 \%$ monomer conversion and the polymer molecular weight was determined by THF GPC: $M_{\mathrm{n}, \mathrm{GPC}}=3.10 \cdot 10^{5}$, and $M_{\mathrm{w}} / M_{\mathrm{n}}=1.24$. The degree of polymerization (DP) was calculated from the calibration curve $\left(M_{\mathrm{n}, \mathrm{GPC}}=163.85 \cdot \mathrm{DP}-\right.$ 7000) and determined to be 2000 . The reaction mixture transferred to $100 \mathrm{~mL}$ pre-weighted, 
round-bottom flask, then the remaining monomer was removed by flushing air overnight and the polymer was used for the next step without further purification.

Synthesis of PBiBEM 2000 (macroinitiator). A $100 \mathrm{ml}$ round-bottom flask was charged with P(HEMA-TMS $)_{2000}(3.60 \mathrm{~g}, 17.8 \mathrm{mmol}), \mathrm{KF}(1.16 \mathrm{~g}, 19.6 \mathrm{mmol}), \mathrm{DTBP}(0.37 \mathrm{~g}, 1.78 \mathrm{mmol})$, and then dry THF $(50 \mathrm{~mL})$ was added under nitrogen. The reaction mixture was cooled down in an ice bath, followed by the injection of tetrabutylammonium fluoride $(0.18 \mathrm{~mL}, 1.0 \mathrm{M}$ in $\mathrm{THF}$, $0.18 \mathrm{mmol}$ ) and subsequent dropwise addition of $\alpha$-bromoisobutyryl bromide $(4.50 \mathrm{~g}, 2.4 \mathrm{~mL}$. $19.6 \mathrm{mmol}$ ) over the course of $20 \mathrm{~min}$. Upon addition, the reaction mixture was allowed to reach room temperature and was stirred for another $16 \mathrm{~h}$. Afterwards solids were filtered of and the mixture was precipitated into methanol/water (70/30), re-dissolved in chloroform $(50 \mathrm{~mL})$ and passed through the column filled with basic alumina. The product was re-precipitated three times in hexanes and dried overnight under vacuum. Apparent molecular weight determined by THF GPC: $M_{\mathrm{n}, \mathrm{GPC}}=3.16 \cdot 10^{5}$, and $M_{\mathrm{w}} / M_{\mathrm{n}}=1.31$.

Synthesis of polymer bottlebrushes. Four bottlebrush polymers were synthesized by ATRP from the same macroinitiator $\left(\mathrm{PBiBEM}_{2000}\right)$ and varying in DP of poly ( $n$-butyl acrylate) (PBA) side chains (Table 1). A typical procedure for the preparation of Brush-1 is described here: A 10 mL Schlenk flask equipped with a stir bar was charged with macroinitiator PBiBEM$_{2000}(0.196 \mathrm{~g}$, $0.703 \mathrm{mmol}$ of BiBEM groups), $n \mathrm{BA}(10.0 \mathrm{~mL}, 70.2 \mathrm{mmol})$, dNbpy (0.155 g, $0.379 \mathrm{mmol})$, $\mathrm{Cu}^{\mathrm{II}} \mathrm{Br}_{2}(4.7 \mathrm{mg}, 0.0210 \mathrm{mmol})$, and anisole $(1.1 \mathrm{~mL})$. The solution was degassed by three freeze-pump-thaw cycles. During the final cycle $\mathrm{Cu}^{\mathrm{I}} \mathrm{Br}(24.6 \mathrm{mg}, 0.1684 \mathrm{mmol})$ was quickly added to the frozen reaction mixture under nitrogen atmosphere. The flask was sealed, evacuated, back-filled with nitrogen five times, and then immersed in an oil bath thermostated at $70{ }^{\circ} \mathrm{C}$. The polymerization was stopped after $18 \mathrm{~h}$, and the monomer conversion was determined 
by ${ }^{1} \mathrm{H}$ NMR (2.6\%), resulting in the brush polymer with DP 26 of side chains. The polymer was purified by three precipitations from cold methanol, and dried under vacuum at room temperature, to a constant mass. Apparent molecular weight was determined using THF GPC: $M_{\mathrm{n}, \mathrm{GPC}}=1.35 \cdot 10^{6}$, and $M_{\mathrm{w}} / M_{\mathrm{n}}=1.30$.

Table 1. Characterization of the molecular bottlebrushes

\begin{tabular}{cccccccccc}
\hline \multicolumn{9}{c}{ GPC } \\
\hline & $n_{\mathrm{bb}}{ }^{\mathrm{a}}$ & $n_{\mathrm{sc}}{ }^{\mathrm{b}}$ & $M_{\mathrm{n}}{ }^{\mathrm{c}}$ & $M_{\mathrm{w}} / M_{\mathrm{n}}{ }^{\mathrm{d}}$ & $L_{0}(\mathrm{~nm})^{\mathrm{e}}$ & $W(\mathrm{~nm})^{\mathrm{f}}$ & $n_{b b}{ }^{g}$ & $n_{s c}{ }^{h}$ & $n_{s c} / n_{g}{ }^{i}$ \\
\hline Brush-1 & 2000 & 26 & $1.35 \times 10^{6}$ & 1.30 & $371 \pm 10$ & $23 \pm 2$ & 1484 & 40 & 23 \\
Brush-2 & 2000 & 60 & $1.63 \times 10^{6}$ & 1.52 & $354 \pm 8$ & $60 \pm 2$ & 1416 & 112 & 64 \\
Brush-3 & 2000 & 100 & $1.75 \times 10^{6}$ & 1.68 & $369 \pm 8$ & $77 \pm 4$ & 1476 & 148 & 85 \\
Brush-4 & 2000 & 130 & $2.14 \times 10^{6}$ & 1.58 & $366 \pm 9$ & $110 \pm 4$ & 1464 & 222 & 127 \\
\hline
\end{tabular}

${ }^{a}$ Number average DP of the bottlebrush backbone estimated from its apparent molecular weight by GPC. ${ }^{b}$ Apparent number average DP of side chains estimated from monomer conversion. ${ }^{c}$ Apparent number average molecular weight measured by GPC. ${ }^{\mathrm{d}}$ Dispersity determined by GPC.

${ }^{\mathrm{e}}$ Contour length of bottlebrushes adsorbed to mica substrate prior to sonication prepared by the LB technique. ${ }^{\mathrm{f}}$ Width of the bottlebrush adsorbed on mica substrate determined by AFM. ${ }^{\mathrm{g}}$ Number average DP of the bottlebrush backbone calculated from the AFM imaged molecules as $n_{b b}=L_{0} / l_{0}$, where $l_{0}=0.25 \mathrm{~nm}$ - monomer contour length. ${ }^{\mathrm{h}}$ Number average DP of the side chains calculated as $n_{s c}=\alpha W /\left(2 Đ l_{0}\right)$, where $Đ=1.2$ - dispersity of bottlebrush side chains determined by GPC of cleaved side chains, $\alpha=\left(1+\pi r_{T} W / 4 L_{0}\right)$ - geometric correction factor due to the hemispherical shape of bottlebrush macromolecules and $r_{T}=0.9$ - LB mass transfer ratio [31]. ${ }^{\mathrm{i}} n_{g} \cong 1.75$ is the average number of repeat units of the backbone between neighboring side chains estimated from previous synthesis.

\subsection{Sonication experiments}

Each sonication was performed in a three-armed Suslick reaction vessel of $\sim 15 \mathrm{~mL}$ of molecular bottlebrush solution in chloroform at a concentration of $0.1 \mathrm{mg} / \mathrm{ml}$. The solutions were deoxygenated with bubbling $\mathrm{N}_{2}$ for 30 min prior to sonication. The temperature was kept between 6 and $9{ }^{\circ} \mathrm{C}$ in an ice-water bath, and the sonication pulse sequence was set to $1 \mathrm{~s} \mathrm{ON/1 \textrm {s }}$ 
OFF, with a power of $6.8 \mathrm{~W} / \mathrm{cm}^{2}$ working at $20 \mathrm{kHz}$. Aliquots were removed from the vessel at various sonication time intervals for AFM analysis.

\subsection{Langmuir-Blodgett monolayers}

At different sonication times, solutions of the molecular bottlebrushes were extracted from the reactor, and $100 \mu \mathrm{L}$ was deposited onto the surface of water (Milli-Q double-distilled, $\rho=18.2$ $\mathrm{M} \Omega$ )/2-propanol (Sigma-Aldrich, HPLC grade) mixture (0.5 wt. \% of propanol) in a LangmuirBlodgett trough (KSV-5000 instrument equipped with a Wilhelmy plate balance) at room temperature. Propanol was added to reduce the surface energy to prevent backbone scission due to adsorption [16]. The monolayer films were transferred from the air/aqueous interface to freshly cleaved mica substrates at a constant pressure of $0.5 \mathrm{mN} / \mathrm{m}$ for AFM studies.

\subsection{AFM imaging and analysis}

Height images of individual molecules were collected using a multimode Atomic Force Microscopy (Bruker) with a NanoScope V controller in PeakForce QNM mode. We used silicon cantilevers with a resonance frequency of $50-90 \mathrm{kHz}$ and a spring constant of about $0.4 \mathrm{~N} / \mathrm{m}$. Digital images of individual molecules were analyzed using a custom software program developed in-house. The contour length of the bottlebrush backbone was measured directly by AFM given the height contrast due to the desorbed side chains segregated around the backbone. More than 600 molecules were counted to obtain the length distributions of the bottlebrushes, ensuring representative statistics.

\section{Results and Discussion}

\subsection{Molecular imaging of degradation products}


Figure 1a displays images of individual molecules of Brush-3 captured at different stages of the sonication process. Apparently, long bottlebrushes undergo progressive fragmentation to smaller species. Figure $1 \mathrm{~b}$ depicts the time evolution of the bottlebrush length distribution during the sonication process. For example, the average contour length of Brush-3 was $L_{0}=369 \pm 8$ $\mathrm{nm}$ before sonication and then decreased to a limiting length of $L_{\text {lim }}=27 \pm 4 \mathrm{~nm}$ within a total sonication time of 4 hours. The length dispersity of the fractured bottlebrushes initially increased at the beginning of sonication and then followed by a decrease with time, as shown in Figure 1c. This is consistent with previously reported result on ultrasonic degradation of poly(methyl methacrylate) and polystyrene that initially narrow distribution increased in dispersity before narrowing again at long sonication times $[32,33]$. We hypothesize that this increase in dispersity is due to multiple fractures of a given parent bottlebrush during a single bubble collapse; the daughters of the first fragmentation are trapped in the elongational flow and experience increasing strain rates that lead to additional fragmentation. Note that scission of the side chains can be neglected for Brush-1, 2 and 3, since their molecular weight is well below the typical limiting molecular weight (40-50 kDa) under similar experimental conditions [34, 35]. For Brush-4 with side chain DP of 222, the average molecular weight of two PBA side chains is about $57 \mathrm{kDa}$, which may cause their rupture. However, the probability of bond scission along any given side-chain to side-chain path is significantly lower than along the backbone. Due to multiple side chains coupled to a bottlebrush backbone, the drag force along the backbone is larger than along a side-chain path. It is also necessary to point out that recombination of daughter fragments can be neglected due to steric repulsion of densely-grafted side chains. Once the backbone is cleaved, the generated radicals will be spontaneously shielded by the side chains, which form a hemispherical cap at the bottlebrush ends hindering the recombination. 

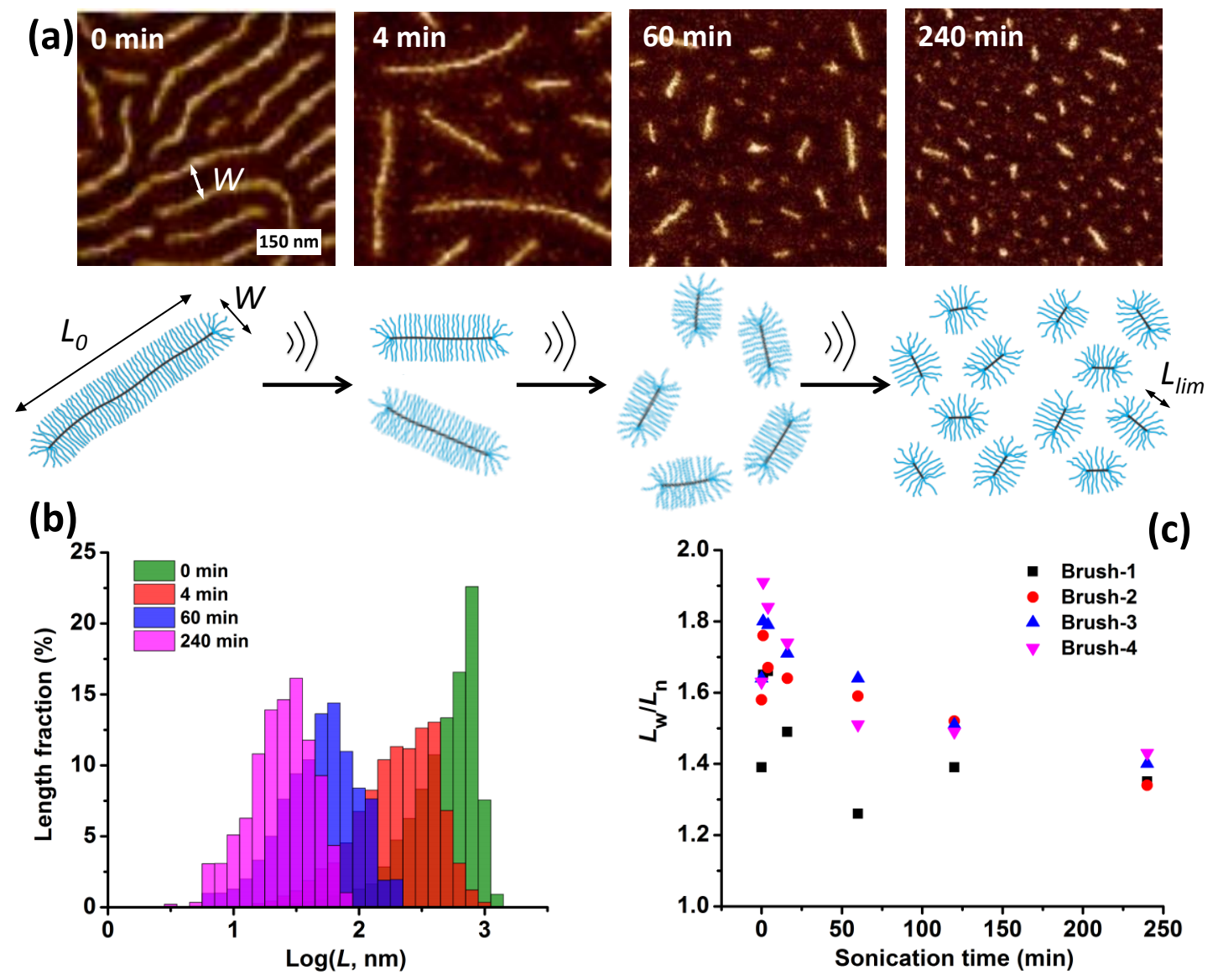

Figure 1. (a) AFM height micrographs of Brush-3 and cartoon displaying fragmentation of bottlebrushes during sonication. (b) The corresponding length distributions and (c) Dependence of the length dispersity, i.e. ratio of weight average contour length $\left(L_{\mathrm{w}}\right)$ to number average contour length $\left(L_{\mathrm{n}}\right)$, with sonication time.

\subsection{Degradation kinetics of molecular bottlebrushes}

The scission rate and the limiting molecular weight for linear polymers can be determined by using the equation derived by Madras et al. [36, 37]. This equation is based on the assumption that sonication-induced cleavage of polymers occurs at the mid-point of the chain and is a firstorder reaction:

$$
\ln \left(\frac{L_{l i m}^{-1}-L_{t}^{-1}}{L_{l i m}^{-1}-L_{0}^{-1}}\right)=-k L_{l i m} t
$$


where $L_{l i m}, L_{0}$ and $L_{t}$ are the limiting number average contour length, the initial number average contour length and the number average contour length of the backbone at time $t$ respectively, and $k$ is the rate constant that is independent of $L_{t}$. Note that Eq. 1 is expressed in terms of bottlebrush contour length, which is linearly proportional to the corresponding molecular weight $\left(M_{i} \sim L_{i}\right)$ typically used in the kinetics analysis $[28,29]$. We use Eq. 1 to fit the experimental data with $L_{l i m}$ and $k$ as the fitting parameters for all four bottlebrush samples. As shown in Figure 2a, the fitted decay profiles (solid curves) are in good agreement with the experimental data (points). The fitting results are summarized in Table 2 . The extrapolated $L_{l i m}$ values in Table 2 are verified by molecular imaging of bottlebrushes after long-time sonication for 4 hours (Figure $2 b)$.

(a)

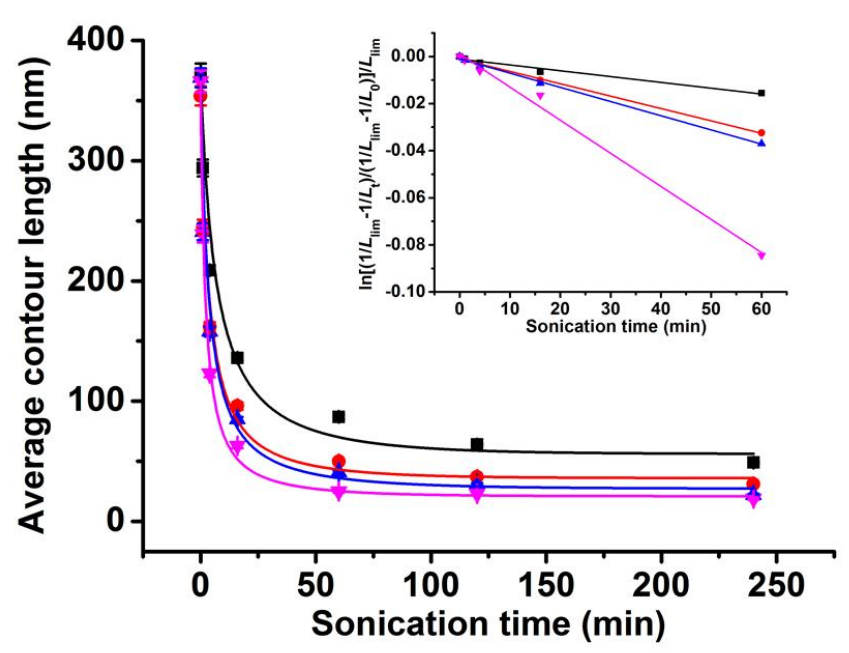

(b)
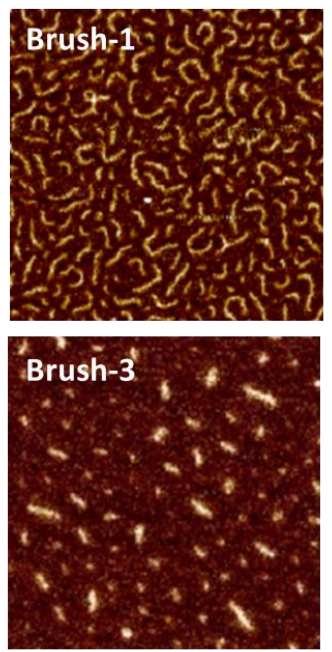
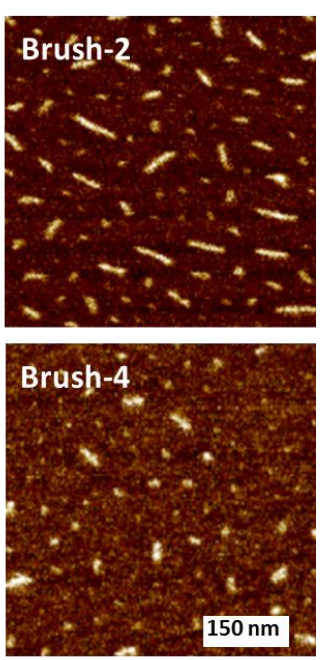

Figure 2. (a) Experimentally determined number average contour length with respect to

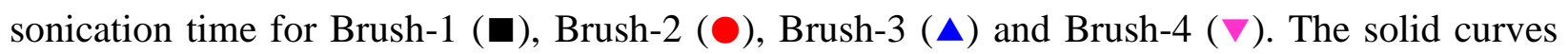
are the corresponding fits using Eq. 1. Inset: The data points are plotted as $\frac{1}{L_{l i m}} \ln \left[\frac{L_{l i m}^{-1}-L_{t}^{-1}}{L_{l i m}^{-1}-L_{0}^{-1}}\right]$ versus time (Eq. 1). (b) AFM height micrographs of the molecular bottlebrushes were captured after sonication for 4 hours. The bottlebrushes with shorter side chains (Brush-1) display longer backbones than the brushes with longer side chains. 
As shown in Table 2, the scission rate increases with increasing side chain DP, while the limiting contour length $\left(L_{\text {lim }}\right)$ of the molecular bottlebrush decreases with increasing side chain DP. Both observations are consistent with previously reported results of sonication-induced scission of poly (alkyl methacrylate) that the scission rate increases and the limiting chain length decreases with increasing bulk size of the alkyl substituent $[34,35]$. This may be explained by larger drag force generated along the bottlebrush backbone with longer side chains during bubble collapse. Note that the limiting contour lengths for all four bottlebrushes are substantially smaller than that for linear polymers under similar sonication conditions. Typical limiting molecular weight for poly (alkyl methacrylates) is about $40 \mathrm{~kg} / \mathrm{mol}$ [35], corresponding to a contour length of $\sim 100 \mathrm{~nm}$, which is about 4 times larger than that for the bottlebrushes with the longest side chains (Brush-4). On the other hand, the densely grafted bottlebrush samples approach their limiting contour length within $\sim 60 \mathrm{~min}$, while it takes hours for linear poly (alkyl methacrylates) to reach their limiting molecular weights under similar conditions (or at even higher sonication powers) [34].

Table 2. Results from fitting analysis in Figure 2.

\begin{tabular}{lcccc}
\hline & $L_{0}(\mathrm{~nm})^{\mathrm{a})}$ & $L_{\text {lim }}(\mathrm{nm})^{\mathrm{b})}$ & $k \cdot 10^{4}\left(\mathrm{~min}^{-1} \cdot \mathrm{nm}^{-1}\right)^{\mathrm{c})}$ & $R_{a d j}^{2}{ }^{\mathrm{d})}$ \\
\hline Brush-1 & $371 \pm 10$ & $56 \pm 6$ & $4.3 \pm 0.8$ & 0.94 \\
Brush-2 & $354 \pm 8$ & $36 \pm 4$ & $7.8 \pm 1.5$ & 0.95 \\
Brush-3 & $369 \pm 8$ & $27 \pm 4$ & $8.2 \pm 1.3$ & 0.96 \\
Brush-4 & $366 \pm 9$ & $21 \pm 2$ & $13.6 \pm 1.3$ & 0.98 \\
\hline a) & Initial contour & length of the bottlo &
\end{tabular}

a) Initial contour length of the bottlebrushes before sonication. ${ }^{\text {b) }}$ Limiting contour length of fractured bottlebrushes. ${ }^{\text {c) }}$ Rate constant of the sonication-induced fracture of bottlebrushes obtained by fitting the experimental data points in Figure 2 a with Eq. $1 .{ }^{\text {d) }}$ The adjusted $R^{2}$ values close to unity demonstrate the goodness of the fit.

\subsection{Data analysis}


For scaling analysis of the dependence of the limiting contour length on the side chain DP, we will consider two different models to calculate the drag force at the center of a bottlebrush backbone. In the first model, solvent is assumed to be freely draining through the side chains as they move (Rouse model); while in the other model, we assume that the side chain drags the solvent in their pervaded volume as they move (Zimm model) [38].

Rouse model. We first consider the force at the center of a chain (maximum force $f_{m}$ ) for linear polymers with $n$ monomers in the elongational flow generated by sonication. Assuming that the chain is fully extended, we can write [39]

$$
f_{m} \sim \zeta_{m} L^{2}
$$

where $L \sim n$ is the contour length, $\zeta_{m}$ is the monomeric friction coefficient.

In this model, it is assumed that solvent can be freely drained through the volume occupied by the side chains as they move. The friction coefficient of each Kuhn monomer is evaluated as $\zeta_{m}=\eta b$, where $b \cong 1.8 \mathrm{~nm}$ is the Kuhn length of PBA side chains and $\eta$ is the viscosity of the solvent. Therefore the total friction coefficient of the whole side chain is the sum of the contributions of each monomer:

$$
\zeta_{R}=N_{s c} \zeta_{m}=N_{s c} b \eta .
$$

where $N_{s c}$ is the number of Kuhn monomers of a side chain. Considering each side chain is an effective monomeric unit of bottlebrush molecules, we can obtain the maximum drag force at the center of the bottlebrush backbone as

$$
f_{m} \sim \zeta_{R} L^{2} \sim N_{s c} L^{2}
$$

As the force along the bottlebrush backbone increases, the rate of backbone scission increases. It is known that the intrinsic tension in the bottlebrush backbone (prior to sonication) is on the 
order of $10 \mathrm{pN}$ [15], while it requires $\mathrm{nN}$ forces to break covalent bonds on the $\sim \mu$ s time scale of a bubble collapse in the sonication experiment [40]. Therefore, the intrinsic tension can be neglected, and the external critical force $\left(f_{c}\right)$ to break the $\mathrm{C}-\mathrm{C}$ bonds in the backbone can be considered as the same for the four bottlebrushes regardless of the side chain DP. This differs from the case of solid filaments that exhibit an increase of the critical force with increasing filament diameter. The backbone will effectively stop breaking once $f_{m}$ becomes smaller than $f_{c}$, and therefore we can obtain the following relation between the limiting contour length $\left(L_{l i m}\right)$ and the number of Kuhn monomers in the side chain $\left(N_{s c}\right)$ :

$$
\begin{gathered}
f_{c} \sim N_{s c} L_{\text {lim }}^{2} \\
L_{\text {lim }} \sim\left(1 / N_{s c}\right)^{1 / 2} .
\end{gathered}
$$

To verify this prediction, we plot the experimentally determined limiting contour lengths as a function $1 / N_{s c}$ (Figure 3 ). The number of Kuhn monomers can be estimated as $N_{s c} \cong$ $\left(n_{s c} / n_{g}\right) l_{0} / b$, where the number average DP of the side chains was determined from the brush width by the AFM-LB method (Table 1) $[9,15,31]$. For our bottlebrush macromolecules, $N_{s c}$ ranges from 3 to 18 . The slope of $0.57 \pm 0.06$ is slightly larger than 0.5 - the scaling exponent predicted by the Rouse model (Eq. 6). This deviation may be attributed to incomplete extension of the bottlebrush backbone in the elongational flow created by bubble collapse. 


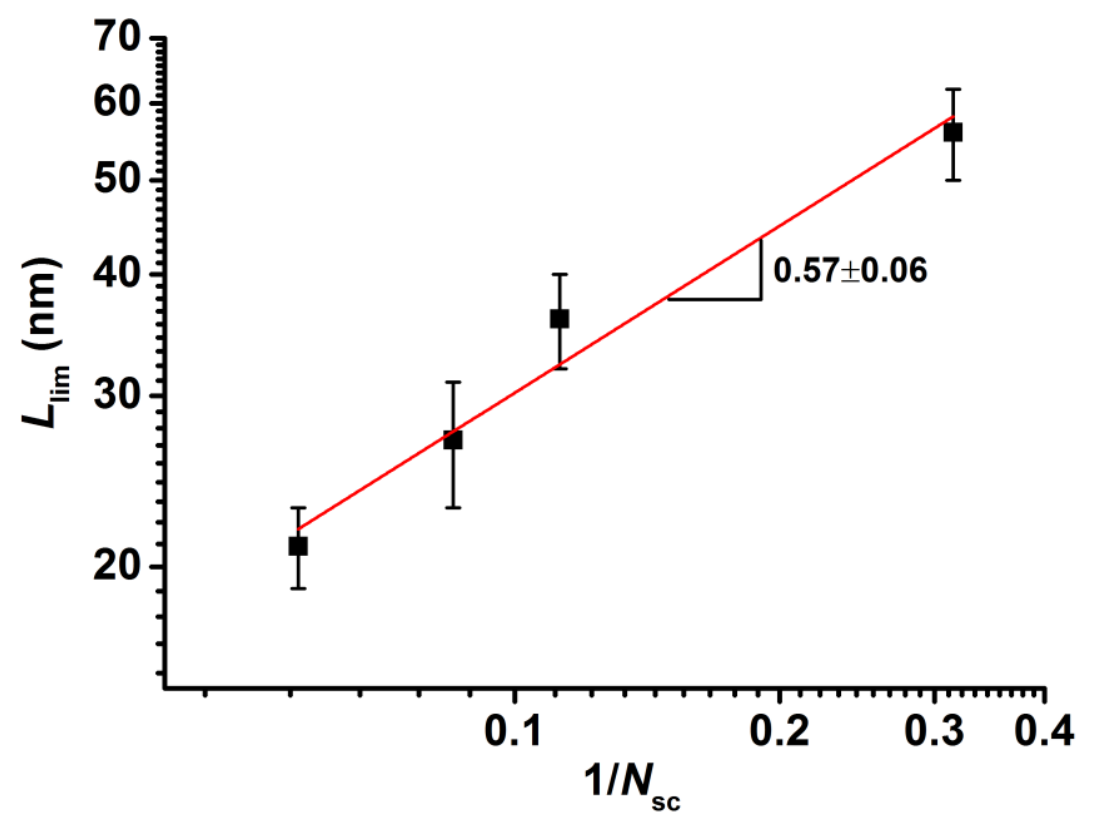

Figure 3. Experimentally determined scaling relation between the limiting contour length $\left(L_{\text {lim }}\right)$ and the number of Kuhn monomers in the side chain $\left(N_{s c}\right)$. The solid line represents the powerlaw fit to the data points.

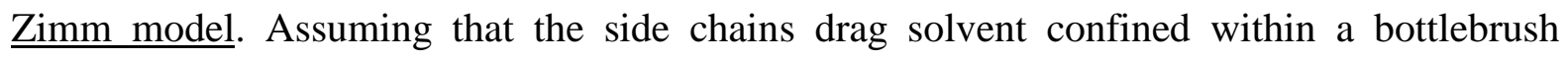
macromolecule, we consider molecular bottlebrushes as solid spherocylinders with radius $R=b N_{s c}^{1 / 2}$ (unperturbed side chains that are smaller than the thermal blob). The aspect ratio of the cylindrical brushes is given as

$$
p=L^{\prime} / 2 R=(L+2 R) / 2 R=L /\left(2 b N_{s c}^{1 / 2}\right)+1
$$

where $L^{\prime}=L+2 R$ is the total length of molecular bottlebrushes including the end caps formed by side chains in solution. The aspect ratios for the four molecular bottlebrushes at limiting backbone contour length $L_{\text {lim }}$ are in the range of $2.4-9.7$. Taking this into consideration, we can express the friction coefficient parallel to the backbone using the equation with numerical correction proposed by Aragon and Flamik for $1 \leq p<\infty$ [41]:

$$
\zeta_{Z}=4 \pi \eta L^{\prime} /[2 \ln (p)+C]
$$


where $\quad C=-0.113192-1.30429 \cdot p^{-0.25}+1.19032 \cdot p^{-0.5}+3.12756 \cdot p^{-1}-1.56699$. $p^{-2}-0.930791 \cdot p^{-2} \cdot \ln (p)$ is the numerical correction.

To derive the forces in the bottlebrush backbone caused by velocity gradient along the backbone, we express the relative velocity $(V)$ as a function of the distance $(x)$ from the middle of the backbone by $V(x)=\dot{\varepsilon} \cdot x$, where $\dot{\varepsilon}$ is the strain rate. The force per unit length along the backbone is given by

$$
f(x)=\zeta_{Z} V(x) / L^{\prime}=4 \pi \eta \dot{\varepsilon} x /[2 \ln (p)+C]
$$

To calculate the maximum drag force at the backbone center $f_{m}$, we need to integrate the absolute value of $f(x)$ from 0 to $L^{\prime} / 2$ and obtain

$$
f_{m}=\frac{1}{2} \pi \eta \dot{\varepsilon} L^{\prime 2} /[2 \ln (p)+C]
$$

Similarly, we can obtain the following scaling relations:

$$
\begin{aligned}
& f_{c} \sim L_{\text {lim }}^{\prime 2} /\left[2 \ln \left(p_{\text {lim }}\right)+C_{\text {lim }}\right] \\
& L_{\text {lim }}^{\prime} \sim\left[2 \ln \left(p_{\text {lim }}\right)+C_{\text {lim }}\right]^{1 / 2}
\end{aligned}
$$

where $p_{\text {lim }}$ and $C_{\text {lim }}$ are the corresponding aspect ratio (Eq. 7) and numerical correction as a function of the limiting contour length (Eq. 8), respectively. Figure 4 shows a log-log plot of $L^{\prime}{ }_{\text {lim }}$ as a function $2 \ln \left(p_{\text {lim }}\right)+C_{\text {lim }}$ with a slope of $0.83 \pm 0.05$, which is markedly larger than the predicted value of 0.5 from Eq. 12 . 


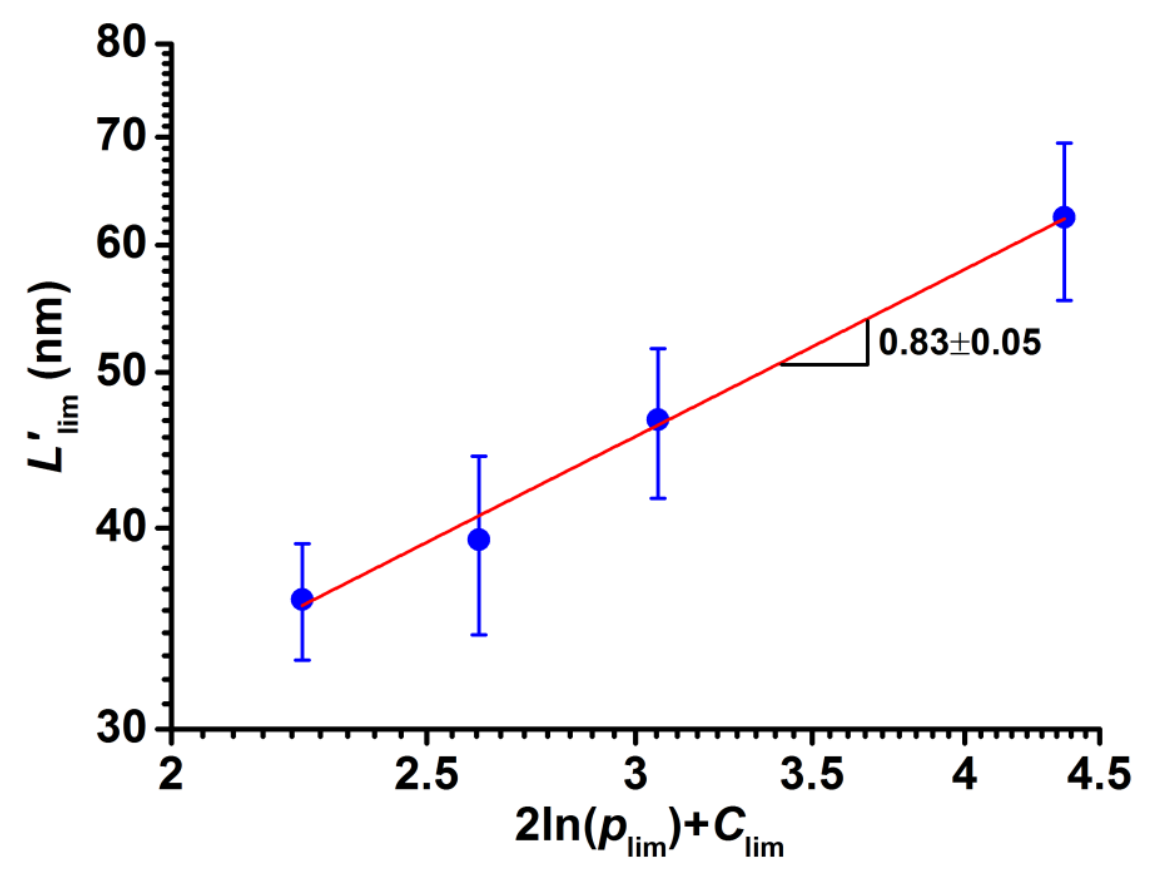

Figure 4. Dependence of the limiting total length of cylindrical bottlebrushes $L^{\prime}$ lim as a function of parameter $2 \ln \left(p_{\text {lim }}\right) /+C_{\text {lim }}$.

\subsection{Discussion}

Scaling analysis of the bottlebrush backbone scission dynamics has been performed to quantify the effect of side chain DP on the limiting contour length using Rouse and Zimm models. The former model shows a better agreement between model predictions and experimental results. This indicates that the solvent molecules drain through the bottlebrush corona producing a larger drag force. The solvent draining can be rationalized by comparing the characteristic relaxation time of the side chains and the shear strain rate generated upon bubble collapse. For the solvent to move through the side chains its flow should be faster than the side chain Zimm relaxation time $\tau_{Z}\left(\dot{\varepsilon}>\tau_{Z}^{-1}\right)$. We can estimate the Zimm relaxation time for the side chains with size $R \cong b N_{s c}^{1 / 2}$ as

$$
\tau_{Z} \cong \tau_{0} N_{s c}^{3 / 2}
$$


where $\tau_{0} \approx \eta b^{3} / k_{B} T \cong 1.0 n s$ is the relaxation time of Kuhn monomer $(b=1.8 \mathrm{~nm})$ in chloroform with viscosity $\eta \sim 0.64 \mathrm{mPa} \cdot s$ at the median temperature $\left(6-9{ }^{\circ} \mathrm{C}\right)$ of $T \cong 281 \mathrm{~K}$ and $k_{B}$ is the Boltzmann constant. From Eq. 13, we can estimate the Zimm relaxation time to change between $6 \mathrm{~ns}$ (Brush-1) and $74 \mathrm{~ns}$ (Brush-4). It is known that the strain rate and, hence, drag force decrease with the distance from an imploding microbubble [18]. Yet, it may be strong enough to induce bond scission, provided that the bottlebrushes are sufficiently long. At the initial contour length $L_{0} \cong 370 \mathrm{~nm}$ (Table 2), the maximum drag force $f_{m} \sim \dot{\varepsilon} L_{\text {lim }}^{2}$ can reach the critical force $f_{c}$ at a lower strain rate, e.g., $2 \times 10^{7} s^{-1}$ for Brush-1 and $3 \times 10^{6} s^{-1}$ for Brush-4. This is slower than the Zimm relaxation $\left(\dot{\varepsilon}<\tau_{Z}^{-1}\right)$, indicating that the bottlebrush dynamics corresponds to non-draining regime described by the Zimm model. However, at a limiting contour length (analyzed in this paper), the bottlebrushes need to reach the vicinity of an imploding microbubble where the strain rate is the highest $\left(\dot{\varepsilon} \cong 10^{9} S^{-1}\right)[18,19,42]$ to achieve the greatest force at the backbone center. In this case, the strain rate is larger than the Zimm relaxation time $\left(\dot{\varepsilon}>\tau_{Z}^{-1}\right)$, suggesting that the bottlebrush dynamics corresponds to draining regime described by the Rouse model. In other words, the bottlebrush dynamics undergoes a transition from non-draining (Zimm) to draining (Rouse) regime as the bottlebrushes become shorter. At the initial stages of sonication, long bottlebrushes $\left(\dot{\varepsilon}<\tau_{Z}^{-1}\right)$ may behave like solid cylindrically shaped objects, similar to carbon nanotubes, nanowires, polymeric fibrils $[18,19$, 25, 27, 28]; while at the later stages, the backbone scission is controlled by solvent draining, which differentiates short bottlebrushes $\left(\dot{\varepsilon}>\tau_{Z}^{-1}\right)$ from solid cylindrical objects. Besides, the critical force for cleavage for solid cylindrically shaped objects depends on their cross-sectional area, in contrast to its independence on diameter (DP of side chains) for bottlebrushes and that it is determined by the strength of backbone bonds. 


\section{Conclusions}

We have studied the effect of the side chain DP on the sonication-induced scission of molecular bottlebrushes. The scission rate has been shown to increase with increasing side chain DP, while the limiting contour length decreases as side chain DP increases. This decrease of the limiting contour length is attributed to higher drag force exerted on the backbone with longer side chains by the solvent flow generated by bubble collapse. To quantify the effect of side chain DP on the limiting contour length, we have performed scaling analysis of the bottlebrush backbone scission dynamics using Rouse and Zimm models. The former model (Rouse) shows a better agreement between model predictions and experimental results, which indicates that flexible and hairy molecular bottlebrushes behave differently from solid cylindrically shaped objects with respect to their interaction with solvent in the sonication process when the bottlebrushes are cut short enough $\left(\dot{\varepsilon}>\tau_{Z}^{-1}\right)$ : solvent drains through the bottlebrush corona formed by side chains, while it cannot penetrate through solid objects.

\section{Acknowledgement}

We gratefully acknowledge funding from the National Science Foundation (DMR 0906985, DMR 1501324, DMR 1122483), DMR 1409710 and Department of the Army (59646-CH).

\section{References}

[1] Sheiko SS, Sumerlin BS, and Matyjaszewski K. Prog Polym Sci 2008;33:759-785.

[2] Lee H-I, Pietrasik J, Sheiko SS, and Matyjaszewski K. Prog Polym Sci 2010;35:24-44.

[3] Pakula T, Zhang Y, Matyjaszewski K, Lee H-I, Boerner H, Qin S, and Berry GC. Polymer 2006;47:7198-7206.

[4] Mpoukouvalas A, Li W, Graf R, Koynov K, and Matyjaszewski K. ACS Macro Lett 2012;2:23-26.

[5] Mukumoto K, Averick SE, Park S, Nese A, Mpoukouvalas A, Zeng Y, Koynov K, Leduc PR, and Matyjaszewski K. Macromolecules 2014.

[6] Daniel WFM, Burdyńska J, Vatankhah-Varnosfaderani M, Matyjaszewski K, Paturej J, Rubinstein M, Dobrynin AV, and Sheiko SS. Nat Mater;Accepted. 
[7] Rzayev J. ACS Macro Lett 2012;1:1146-1149.

[8] Wu D, Nese A, Pietrasik J, Liang Y, He H, Kruk M, Huang L, Kowalewski T, and Matyjaszewski K. ACS Nano 2012;6:6208-6214.

[9] Sheiko SS, Sun FC, Randall A, Shirvanyants D, Rubinstein M, Lee H-I, and Matyjaszewski K. Nature 2006;440:191-194.

[10] Park I, Sheiko SS, Nese A, and Matyjaszewski K. Macromolecules 2009;42:1805-1807.

[11] Lebedeva NV, Nese A, Sun FC, Matyjaszewski K, and Sheiko SS. Proc Natl Acad Sci U S A 2012;109:9276-9280.

[12] Li Y, Nese A, Matyjaszewski K, and Sheiko SS. Macromolecules 2013;46:7196-7201.

[13] Li Y, Nese A, Hu X, Lebedeva NV, LaJoie TW, Burdyńska J, Stefan MC, You W, Yang W, Matyjaszewski K, and Sheiko SS. ACS Macro Lett 2014;3:738-742.

[14] Panyukov SV, Sheiko SS, and Rubinstein M. Phys Rev Lett 2009;102:148301.

[15] Panyukov S, Zhulina EB, Sheiko SS, Randall GC, Brock J, and Rubinstein M. J Phys Chem B 2009;113:3750-3768.

[16] Lebedeva NV, Sun FC, Lee H-I, Matyjaszewski K, and Sheiko SS. J Am Chem Soc 2008;130:4228-4229.

[17] Li Y, Nese A, Lebedeva NV, Davis T, Matyjaszewski K, and Sheiko SS. J Am Chem Soc 2011;133:17479-17484.

[18] Hennrich F, Krupke R, Arnold K, Rojas Stütz JA, Lebedkin S, Koch T, Schimmel T, and Kappes MM. J Phys Chem B 2007;111:1932-1937.

[19] Huang YY, Knowles TPJ, and Terentjev EM. Adv Mater 2009;21:3945-3948.

[20] Lucas A, Zakri C, Maugey M, Pasquali M, Schoot Pvd, and Poulin P. J Phys Chem C 2009;113:20599-20605.

[21] Naumov AV, Tsyboulski DA, Bachilo SM, and Weisman RB. Chem Phys 2013;422:255263.

[22] Wang X, Guerin G, Wang H, Wang Y, Manners I, and Winnik MA. Science 2007;317:644-647.

[23] Guérin Gr, Wang H, Manners I, and Winnik MA. J Am Chem Soc 2008;130:1476314771.

[24] Gilroy JB, Gädt T, Whittell GR, Chabanne L, Mitchels JM, Richardson RM, Winnik MA, and Manners I. Nat Chem 2010;2:566-570.

[25] Sawawi M, Wang TY, Nisbet DR, and Simon GP. Polymer 2013;54:4237-4252.

[26] Kim HK and Matyjaszewski K. J Am Chem Soc 1988;110:3321-3323.

[27] Saito T, Kuramae R, Wohlert J, Berglund LA, and Isogai A. Biomacromolecules 2012;14:248-253.

[28] Tang S, Vongehr S, Wan N, and Meng X. Mater Chem Phys 2013;142:17-26.

[29] Church DC, Peterson GI, and Boydston AJ. ACS Macro Lett 2014;3:648-651.

[30] Sheiko SS, da Silva M, Shirvaniants D, LaRue I, Prokhorova S, Moeller M, Beers K, and Matyjaszewski K. J Am Chem Soc 2003;125:6725-6728.

[31] Burdyńska J, Daniel W, Li Y, Robertson B, Sheiko SS, and Matyjaszewski K. Macromolecules 2015;48:4813-4822.

[32] Wu CB, Sheth PJ, and Johnson JF. Polymer 1977;18:822-824.

[33] Madras G, Kumar S, and Chattopadhyay S. Polym Degrad Stab 2000;69:73-78.

[34] Malhotra SL. J Macromol Sci, Pure Appl Chem 1986;23:729-748.

[35] Daraboina N and Madras G. Ultrason Sonochem 2009;16:273-279.

[36] Chattopadhyay S and Madras G. J Appl Polym Sci 2003;88:2818-2822. 
[37] Vijayalakshmi SP and Madras G. J Appl Polym Sci 2006;100:4888-4892.

[38] Rubinstein M and Colby RH. Polymer Physics. Oxford: Oxford University Press, 2003.

[39] Odell JA and Keller A. J Polym Sci, Part B: Polym Phys 1986;24:1889-1916.

[40] Grandbois M, Beyer M, Rief M, Clausen-Schaumann H, and Gaub HE. Science 1999;283:1727-1730.

[41] Aragon SR and Flamik D. Macromolecules 2009;42:6290-6299.

[42] Pagani G, Green MJ, Poulin P, and Pasquali M. Proc Natl Acad Sci USA 2012.

\section{Table of Contents (TOC)}

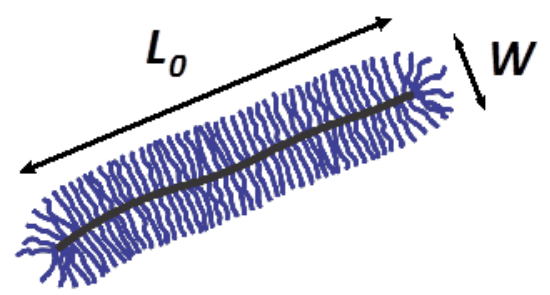

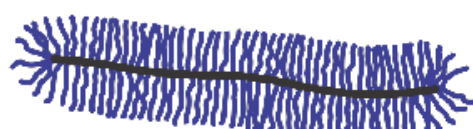

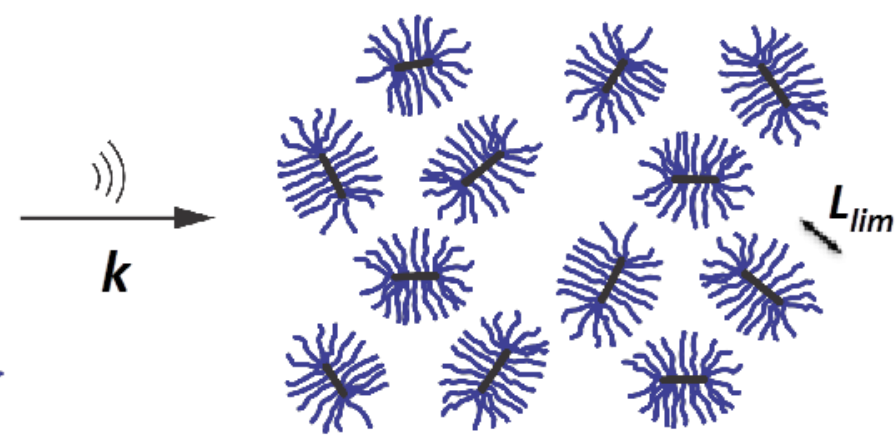

$$
L_{0,1}=L_{0,2} \quad W_{1}<W_{2} \longrightarrow L_{\text {lim, } 1}>L_{\text {lim,2 }} \quad k_{1}<k_{2}
$$




\section{Sonication-Induced Scission of Molecular Bottlebrushes:}

\section{Implications of the "Hairy" Architecture}

Yuanchao Li ${ }^{\text {a }}$, Zhenbin Niu ${ }^{b}$, Joanna Burdyńska ${ }^{c}$, Alper Nese ${ }^{c}$, Yang Zhou ${ }^{a}$, Zachary S. Kean b, Andrey V. Dobrynin ${ }^{d}$, Krzysztof Matyjaszewski ${ }^{\text {, }}$, Stephen L. Craig ${ }^{\mathrm{b}}$, Sergei S. Sheiko a,*

a Department of Chemistry, University of North Carolina, Chapel Hill, North Carolina 27599, United States

${ }^{\mathrm{b}}$ Department of Chemistry, Duke University, Durham, North Carolina 27708, United States

${ }^{\mathrm{c}}$ Department of Chemistry, Carnegie Mellon University, Pittsburgh, Pennsylvania 15213, United States

${ }^{\mathrm{d}}$ Department of Polymer Science, University of Akron, Akron, Ohio 44325, United States

* Correspondence to: Sergei S. Sheiko (Email: sergei@email.unc.edu)

Table of Contents (TOC)

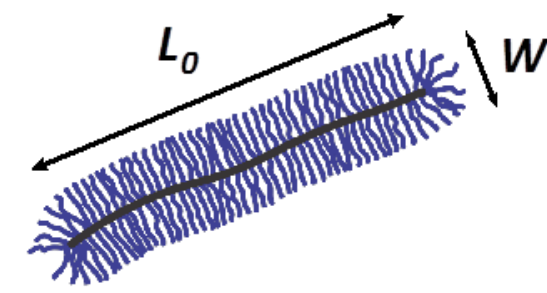

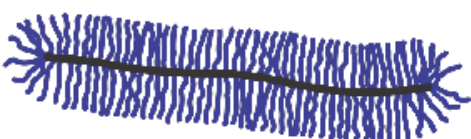
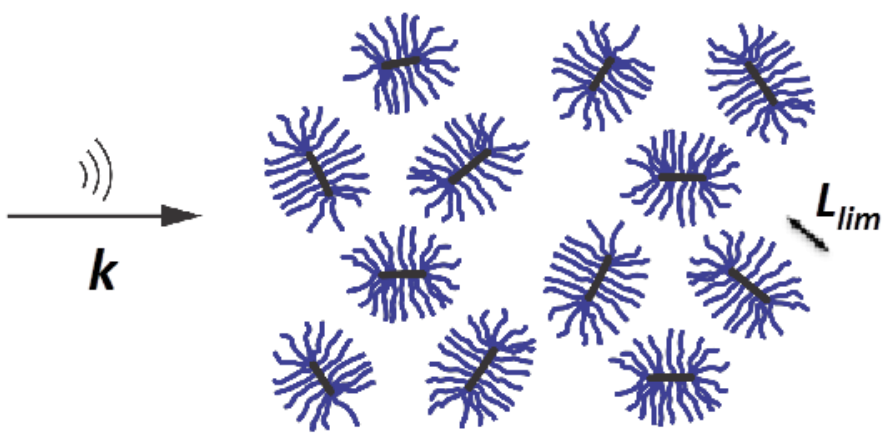

$L_{0,1}=L_{0,2} \quad W_{1}<W_{2} \longrightarrow L_{\text {lim }, 1}>L_{\text {lim,2 }} \quad k_{1}<k_{2}$ 